\title{
Observational survey of the puzzling star HD 179821: Photometric variations and period analysis ${ }^{\star}$
}

\author{
H. Le Coroller ${ }^{1}$, A. Lèbre ${ }^{2}$, D. Gillet ${ }^{1}$, and E. Chapellier ${ }^{3}$ \\ 1 Observatoire de Haute-Provence (CNRS), 04870 Saint-Michel-l'Observatoire, France \\ ${ }^{2}$ Groupe de Recherche en Astronomie et Astrophysique du Languedoc-UMR 5024/ISTEEM (CNRS), \\ Université Montpellier II, Place E. Bataillon, 34095 Montpellier Cedex 05, France \\ 3 Observatoire de la Côte d'Azur, Département Fresnel, UMR 6528, BP 4229, 06304 Nice Cedex 4, France
}

Received 8 August 2002 / Accepted 19 December 2002

\begin{abstract}
From new photometric observations ( $U B V R I)$, we present the characteristic features of the light variations of the evolved star HD 179821 (= SAO 124414 = IRAS 19114+0002). Our data, collected through 1999 and 2000, have been combined together with previous photometric measurements available in the literature. Thus, a long term $V$-light curve (gathering more than 10 years of observations for HD 179821) has been composed. We have analysed it with the Fourier transform method. Two main frequencies are present in the resulting power spectrum, reflecting a dominant bimodal pulsator behavior. A long term phenomenon is also found, but it is not possible to decide whether it is periodic. The Fourier analysis has also been applied on two other filters $(U$ and $B)$ and confirms the detected frequencies. On the basis of our period analysis, we discuss the nature of HD 179821: low-mass post-AGB star or high-mass star.
\end{abstract}

Key words. stars: atmospheres - stars: AGB and post-AGB - shock waves - stars: oscillations - stars: fundamental parameters - stars: individual: HD 179821

\section{Introduction}

HD 179821 (= SAO 124414 = IRAS 19114+0002 = AFGL $2343=$ V1427 Aql = HIP 94496) is an oxygen-rich evolved star surrounded by an extended envelope of gas and dust. This object is still the subject of debate to know whether it is an intermediate-mass post-AGB star or a massive supergiant.

One way to classify this kind of object is to study its chemical composition and/or the characteristic features of the shell surrounding the star. Indeed, the abundances of s-elements synthetized during the third dredge-up of the AGB life are good indicators to decide between a post-AGB or a hypergiant status (Busso et al. 1995; Van Winckel et al. 1996). In HD 179821 however, the s-element abundances are not known with very good accuracy. For instance and depending on the adopted effective temperature for HD 179821, Zacs et al. (1996) and Reddy \& Hrivnak (1999) have found s-elements in over-abundance, while Thèvenin et al. (2000) have obtained a slight deficiency.

On another hand, the expansion velocity of the shell can also help to classify the star. The typical expansion velocity of the shell of post-AGB stars is about $10-20 \mathrm{~km} \mathrm{~s}^{-1}$ while

Send offprint requests to: $\mathrm{H}$. Le Coroller, e-mail: coroller@obs-hp.fr

* based on observations carried out at the Observatoire de Haute Provence, France, operated by the Centre National de la Recherche Scientifique (CNRS). the large outflow velocity of HD $179821\left(34 \pm 2 \mathrm{~km} \mathrm{~s}^{-1}\right.$, Zuckerman \& Dyck 1986) would be rather in favor of the massive hypothesis. However Josselin \& Lèbre (2001) have given an example of another post-AGB object $(\mathrm{OH} 231.8+4.2)$ with such a large outflow velocity value $\left(30 \mathrm{~km} \mathrm{~s}^{-1}\right)$. From radio observations, these authors have also derived a very low value for the ${ }^{12} \mathrm{C} /{ }^{13} \mathrm{C}$ ratio which is not common for massive stars.

The distance and consequently the luminosity of the star is another argument to help in its classification. If HD 179821 lies at $1 \mathrm{kpc}$, it is clearly a post-AGB star while if it is located at $6 \mathrm{kpc}$, its luminosity thus being larger, its initial mass is consequently over $20 M_{\odot}$. Nevertheless, the distance of HD 179821 is not well determined by the trigonometric parallax given by Hipparcos. Consequently, an indirect way must be used to estimate this distance. Zuckerman \& Dyck (1986) have noted that the high LSR velocity of HD $179821\left(105 \pm 1 \mathrm{~km} \mathrm{~s}^{-1}\right)$ would place this star at $6 \mathrm{kpc}$. Jura \& Werner (1999) have also claimed that the $\mathrm{OH}$-maser ring is best understood if HD 179821 lies at $6 \mathrm{kpc}$. Indeed, at $1 \mathrm{kpc}$ the ultraviolet dust opacity deduced from the $\mathrm{OH}$-radius is too large. Moreover, using a radiative transfer model of the nebula, Hawkins et al. (1995) have obtained a good fit to the observed inner and outer radius of the shell and to its spectral energy distribution, if HD 179821 lies at a distance of about $6 \mathrm{kpc}$. But for this distance, the height of HD 179821 above the galactic plane would be around 524 pc (Josselin \& Lèbre 2001) which is quite above 
the scale height for $\geq 20 M_{\odot}$ stars (Miller \& Scalo 1979). Thus, arguments pointing to a large distance and a high mass are still open to criticism (Josselin \& Lèbre 2001). Notably, the distance of $6 \mathrm{kpc}$, deduced from the $V_{\mathrm{lsr}}=105 \mathrm{~km} \mathrm{~s}^{-1}$ (Zuckerman \& Dyck 1986) is not obvious because the star would then be outside the galactic disk.

Very recently Gledhill (Gledhill et al. 2001; Gledhill \& Takami 2001) found some new arguments in favor of the massive status of HD 179821. Notably, these authors show that the $\mathrm{OH}$-shell feature is consistent with a distance estimate to $6 \mathrm{kpc}$. Moreover, they find that the $\mathrm{SiO}$ emission (as the $\mathrm{OH}-$ maser emission) arises in a large-scale detached shell, rather than from a location close to the star. This effect is also observed in a yellow hyper-giant star, IRC +10420 . The connection between these two stars is a strong argument in favor of the hyper-giant status for HD 179821. In the same way, Molster et al. (2002) have concluded that the circumstellar dust shell of HD 179821 is comparable to the ones of two other massive stars: AFGL 4106 and IRC +10420. Notably, for HD 179821, the detected $10 \mu \mathrm{m}$ complex is dominated by the red-shifted amorphous silicate feature and its shape and position strongly resemble to the feature detected in AFGL 4106. Also, the strength of the ratio of the $32.8 \mu \mathrm{m}$ to $33.6 \mu \mathrm{m}$ features suggests that HD 179821 is massive and likely a post-red supergiant.

HD 179821 is one of these puzzling objects where the distinction between low mass post-AGB and massive stars is not easy. Furthermore, both post-AGB and massive evolved stars are statistically rare and their pulsation characteristics are still poorly investigated and not completely understood. To better understand this kind of object, we have undertaken long term observational programs in order to study the variability and the dynamics of the atmosphere of HD 179821. Indeed, pulsation phenomena can help to constrain the stellar parameters, such as mass, luminosity, effective temperature and metallicity. The main goal of this work is to present the photometric observations of HD 179821 and to deduce the photometric amplitude and the pulsation period(s) of this star. This paper is organized as follow: observations and data processing are described in Sect. 2, while period searching by folding light curves and by Fourier transform is presented in Sect. 3. Finally, the status of HD 179821 and conclusions are presented in Sect. 4.

\section{Description of the observations}

From a spectroscopic monitoring with a $2 \mathrm{~m}$-class telescope and the combined analysis of photometric data, Barthès et al. (2000) found a pulsation period of $\sim 37$ days for the typical post-AGB star HD 56126. A previous theoretical work devoted to post-AGB stars (Zalewski 1993) has predicted an average of about 50 days for the pulsation period of these objects. Thus, for HD 179821, we have undertaken a first photometric monitoring roughly covering this duration (2 months, from August to October 1999). But contrary to what we have expected, the resulting light curve (Fig. 1) shows that we have not covered a complete period. Consequently, we have performed a second and longer photometric monitoring, from August to December 2000 .
For all these photometric observations, we used the $80 \mathrm{~cm}$ and the $1.20 \mathrm{~m}$ telescopes at the Observatoire de HauteProvence (OHP, France), in 1999 and in 2000 respectively. In 1999, we observed HD 179821 very regularly (every clear night), while in 2000, we observed the star once a week only but over four months. In both cases we performed differential photometry with Cousin filters $B_{\mathrm{c}}, V_{\mathrm{c}}, R_{\mathrm{c}}, I_{\mathrm{c}}$ and in $U^{\prime}$. The transmission curves of these filters and the CCD characteristics are given on the OHP WEB server (http: //www . obs-hp. fr). Note that we did not work in a standard photometric system. Moreover, in order to spread the flux on the CCD, the images were acquired out of focus. Thus, we increased the exposure time to obtain a good signal-to-noise ratio. The tables with all our measurements (data collected in 1999 and in 2000) are available upon request from H. Le Coroller (coroller@obs-hp.fr).

\subsection{Photometric observations in 1999}

In 1999, we performed on HD 179821 one or two exposures per night in each filter $(U B V R I)$. With the $80 \mathrm{~cm}$ telescope at OHP, the CCD field is $3.6^{\prime} \times 3.6^{\prime}$. In such a reduced field, there was only one reference star (HD 179796) and we could not be sure about its stability. Fortunately, for the observations collected in 2000, the CCD field being larger, it was therefore possible to check the stability of this reference star (see next section). Hence, for the 1999 observations, in each filter, we found the difference in magnitude between HD 179821 and the reference star (HD 179796).

Figure 1 shows the light curves of HD 179821 in the five filters we have used in 1999: $U, B, V, R$ and $I$ (from bottom to top). In order to present these five light curves in the same figure, we have introduced the following magnitude offsets: $\Delta U=0, \Delta B=0.97, \Delta V=1.4, \Delta R=1.55, \Delta I=1.77$. For each light curve, a third order polynomial fit is also displayed. The corresponding rms is about $2 \times 10^{-2}$ for the $U$-light curve, while it is about $8 \times 10^{-3}$ for $B, V, R$ and $I$-light curves.

We can point out that the time of observed maxima of the light curves seem to depend on the filters. The larger the filter wavelength, the earlier the corresponding magnitude curve reaches its maximum. Consequently, we observe a time shift of about seven days between the $U$ and the $I$ extrema. Moreover, when the filter wavelength is increasing from $U V$ to IR, one can notice a strong color effect with a decreasing photometric amplitude. While in the $U$ filter we observe a variation of about $0.25 \mathrm{mag}$, the variation is only of about $0.07 \mathrm{mag}$ in the $I$-filter. This effect can be understood as a strong temperature variation.

Obviously in these data only a part of the characteristic time scale has been covered. Thus we see from Fig. 1 that the characteristic time scale of the expected oscillation is larger than 60 days, i.e, larger than the length of our first observational survey. In order to find a characteristic period for HD 179821, we have performed a second monitoring from August to December 2000. 


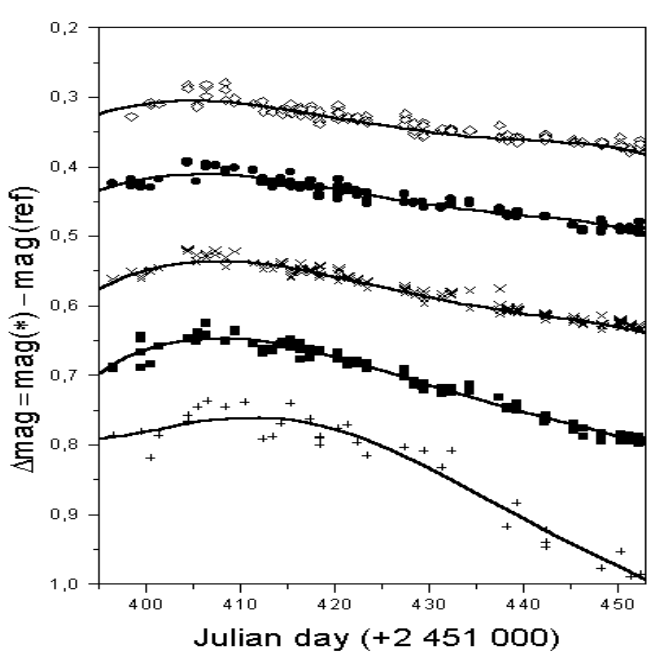

Fig. 1. From bottom to top: $U, B, V, R$ and $I$ magnitude curves of HD 179821 (displayed with magnitude offsets, see text), for the data we have collected from August to October 1999. We have performed differential photometry, using the reference star (HD 179796). For each curve, a third order polynomial fit has been adjusted and its corresponding rms is given in the text.

\subsection{Photometric observations in 2000}

In 2000, we used the $1.20 \mathrm{~m}$ telescope at OHP. Because of its large field of view $\left(11.8^{\prime} \times 11.8^{\prime}\right)$ it was first possible to verify the stability of the reference star HD 179796 (hereafter noted 2) we used for the observations we collected in 1999. Indeed three other reference stars, SAO 124421, GSC 0046303731 and GSC 00463-03582 (hereafter noted 3, 4 and 5, respectively) were also present in the observed field. Figure 2 presents, for the $V$-filter, the stability tests (subtraction of $V$ magnitude) we made on those four reference stars. It clearly shows that, during the observational monitoring performed in 2000, the $V$-magnitude of the star HD 179796 remains stable when compared to the $V$-magnitudes of the three other reference stars (the corresponding rms are indicated in Fig. 2). Outside the standard deviation among the observations (about two percent), all the four reference stars have not presented any light variation during 140 days. Perhaps a very small slope (under one percent over 140 days) might be present. The same kind of result is obtained for the other filters (not presented here).

In 2000, we performed differential photometry, comparing the magnitude of HD 179821 with that of an artificial star resulting from the combination of the four reference stars discussed above. In Fig. 3, we present the light curves of HD 179821 we obtained through this second monitoring (2000) in the five filters $U, B, V, R$ and $I$ (from bottom to top). Moreover, each single point is the average of five consecutive measurements. For convenience we have also introduced magnitude offsets in order to present those five light curves on the same graphic: $\Delta U=0.2, \Delta B=0.45, \Delta V=0.8, \Delta R=0.9$, $\Delta I=1.0$. Finally, for each light curve, a fifth order polynomial fit has been adjusted and the corresponding rms is about $2 \times 10^{-2}, 10^{-2}, 6 \times 10^{-3}, 5 \times 10^{-3}$ and $9 \times 10^{-3}$, for the $U, B$, $V, R$ and $I$ light curves respectively.

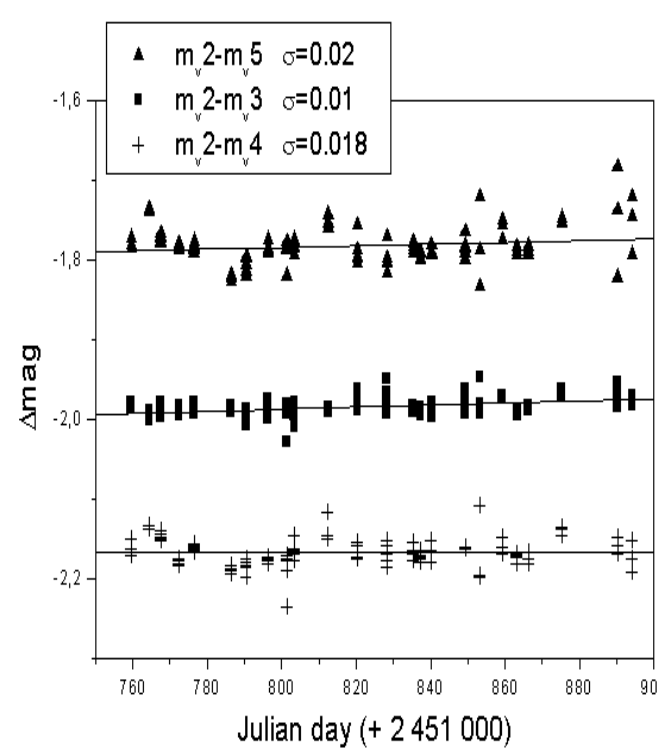

Fig. 2. Subtraction of the $V$ magnitude of the reference star HD 179796 (noted 2) with the $V$ magnitude of three other reference stars observed in 2000 (SAO 124421, GSC 00463-03731, and GSC 00463-03582, noted 3, 4 and 5 respectively). All the four reference stars have not presented any light variation during the 140 days of our monitoring, and the corresponding rms are indicated in the legend frame. For convenience, an offset of -1.1 has been introduced in the $\left(m_{V} 2-m_{V} 4\right)$ data.

For all these light curves, there is about 140 days between the first and the last points (see Fig. 3). Unfortunately, with these observations only, we still cannot be sure that we have covered a whole characteristic time scale. However from these data, we can estimate the amplitude of the light variations for the $U, B$ and $V$ filters (during about 130 days): $\Delta U=0.27$, $\Delta B=0.21, \Delta V=0.16$. For the $R$ and $I$ filters, the light variations we find from a shorter coverage of our observations (during about 100 days), are only rough estimations: $\Delta R=0.10$ and $\Delta I=0.07$. In any case, all these light amplitudes can be considered as minimum limits of the real values. It is important to notice that the strong color effect already mentioned in the data collected in 1999 is still present in the data collected in 2000 (decreasing amplitude with increasing filters from UV to IR).

\section{Period searching}

\subsection{Folding the light curves (1999 \& 2000)}

In order to derive the characteristic time scale of HD 179821, we first combined our two independant sets of data. But this operation appeared to be a very delicate task depending on the considered filter. Indeed the two data sets (1999 and 2000) were not collected with the same instrumentation (although the same filters were used, and the same method applied with the same reference star HD 179796). Hence for example, the characteristic of the two CCDs we have used are slightly different (see the OHP WEB server). However, it turned out that, for the $B$ and $V$-light curves, the two data sets were almost at the same level (less than 1\% magnitude difference between the maxima observed in 1999 and 2000). For these $B$ and $V$-filters, we 


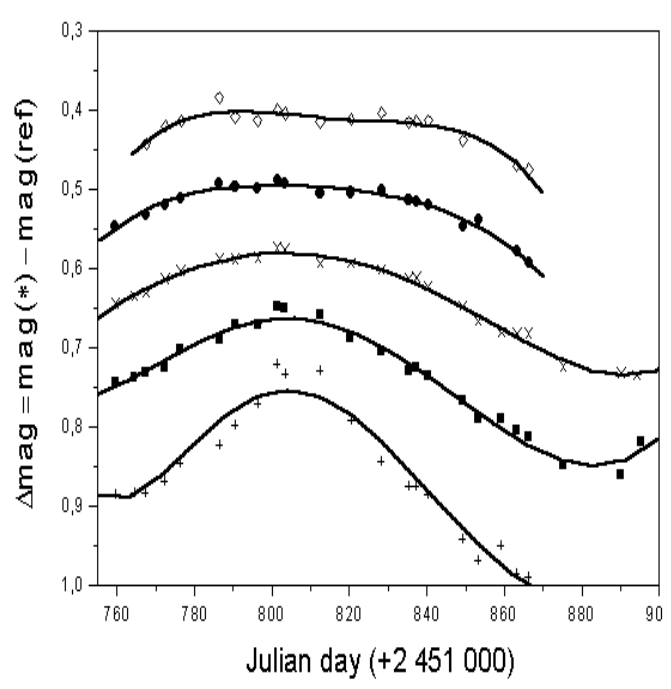

Fig. 3. From bottom to top: $U, B, V, R$ and $I$ magnitude curves of HD 179821 (displayed with magnitude offsets, see text), for the data we collected from August to December 2000. Note that we performed differential photometry, comparing the magnitude of HD 179821 with that of an artificial star composed with four reference stars. A fifth order polynomial fit has been adjusted to each curve, and the corresponding rms are given in the text.

assumed that both photometric systems (in 1999 and in 2000) are in rather good agreement. The consistency of this assumption will be discussed in Sect. 3.2. On the contrary, for the $U$ filter an offset is present when combining the two data sets. This may be due to the difference between the quantum efficiencies of the two CCDs, especially in the UV region. For the $R$ and $I$ filters however, we have not tried to append the two sets of data (1999 and 2000) because the light curves presented too small variations and the duration of the monitoring in these filters was shorter compared to the one in the other filters (especially in 2000). Thus, it appears quite impossible to combine these three sets of data $(U, R$ and $I$ ) with a precision as good as the one we obtained for $B$ and $V$-filters.

Then, once the $B$ and $V$-data (from 1999 and from 2000) have been combined into global light curves, we tried to fold our observations. If we consider that there is only one pulsation cycle between the 1999 and 2000 data, it appears that the best solution in the $B$-filter is found for a period of about 402 days, which is not very consistent with the length we observed between the minimum and the maximum of the $B$ light curve from 2000 ( 80 days, i.e. half a period in the case of a sinusoidal variation, see Fig. 3). Thus, we can expect at least two pulsation cycles between the 1999 and 2000 data. If we consider that there are two pulsation cycles between the 1999 and 2000 data, it appears that the best solution in the $B$-filter is found for a period of $201 \pm 5$ days. Figure 4 displays the corresponding folded $B$-light curve. As shown in Fig. 4 , the $B$-filter light curve obtained in 1999, once translated of $2 \times 201$ days, is well superimposed to the curve obtained in 2000 . The first observed point of the $B$-light curve in 2000 (corresponding to the heliocentric Julian Date HJD = 2451 759.502) has been placed at phase 0.0 for convenience. In the same way, if we consider three cycles between the 1999 and 2000 data, the best solution in $B$-filter is found for a period of $134 \pm 5$ days.
In the $V$-filter, we found almost the same results and the best solution is $P=204 \pm 5$ days for two cycles or $P=136 \pm$ 5 days for three cycles between 1999 and 2000 .

With this method (folding light curves) and the mean of the periods, we found that in $B$ and $V$-filter, 135 days ( \pm 5 days) or 202 days ( \pm 5 days) seem to be roughly the duration of the photometric variation corresponding to our two year survey. But, as we can see in Fig. 4, we have not covered a complete cycle with our data. Moreover, the light curve might be the result of several periods as well, and then we cannot be sure about our method when considering only one period to fold our observations. However, with previous and complementary data available in the literature and offering a long term duration (more than 10 years) for the observation of HD 179821, we have also meticulously looked for its pulsation period(s) with a Fourier transform analysis.

\subsection{Gathering complementary data}

While in 2000 we were collecting the final data for HD 179821, two independant studies became available in the literature, providing for this object new photometric measurements complementary to ours.

First, Arkhipova et al. (2001) published $U B V$ photometric observations of HD 179821, collecting data over about ten years (1990-1999). Their measurements were obtained with a good accuracy ( $\pm 0.005 \mathrm{mag})$ but without precise regularity and only 169 measurements were obtained in each filter. They revealed the semiregular light variations of HD 179821 with the following amplitudes: $\Delta U=0.25, \Delta B=0.15$ and $\Delta V=0.10$. Looking for pulsation period(s), they also performed a Fourier analysis on their observational data in the $B$-filter. Hence, they found a double periodicity in HD $179821\left(P_{1}=205 \pm 2\right.$ days and $P_{2}=142 \pm 2$ days). Besides the variability of HD 179821 likely due to bimodal pulsations, they also detected (from observations covering only 7 years) a trend in its brightness and in its colors indexes. Several possible explanations were investigated (a rise in the stellar temperature, a variable stellar wind, a decrease in the reddening of the circumstellar dust envelope, a stellar companion), while a phenomenon directly related to stellar evolution was rejected. Finally, from 100 year photographic observations of HD 179821, they detected an increasing but fluctuating visual brightness, attributed to a rapid evolutionary phase (like the post-AGB phase).

On another hand, in a general study devoted to variability for a sample of about 40 proto-planetary nebulae, Hrivnak et al. (2001) published a differential $V$-light curve of HD 179821 covering almost 7 years (1994-2000). From this set of data, Hrivnak et al. (2001) did not infer any period.

The final part of the observations discussed by Arkhipova et al. (2001) were collected in 1999, while, at the same time, we were observing HD 179821 with the $80 \mathrm{~cm}$ telescope at OHP. Hence, our own observations (about 170 measurements between 1999 and 2000, in each filters) appeared to be complementary (in the $U B V$ filters at least). Moreover, Arkhipova et al. (2001) also performed differential photometry (in $U, B$ and $V$ ) on HD 179821, using HD 179796 as a reference star. 


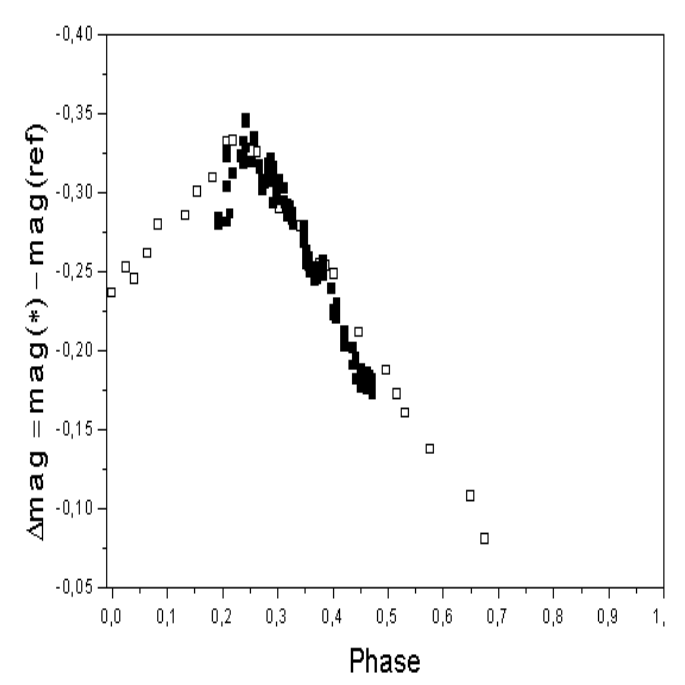

Fig. 4. Folded $B$-filter magnitude curve. Open squares are data from 2000, while filled symbols are data from 1999 translated of $2 \times$ 201 days. Phase 0.0 corresponds to $\mathrm{HJD}=2451759.502$ i.e., to the position of the first observed point of the B light curve in the data collected in 2000 .

Thus, using the few measurements collected at the same dates as calibrators, we could easily merge these two series of data into larger basetime files. We also decided to numerically extract Hrivnak's $V$-data because they were time coincident with $V$-data from Arkhipova et al. (2001), and also with some of our own $V$-measurements (collected in 1999 and in 2000). Finally, we have also taken all the available $V$-HIPPARCOS measurements of HD 179821 that appeared to be prior and/or time coincident to the first $V$-data from Arkhipova et al. (2001). We merged all those $V$-data, in order to compose a $V$-light curve of HD 179821 covering a very long basetime. Figure 5 presents the final $V$-light curve of HD 179821, resulting from the merging of these observational data sets. Note that each point in the HIPPARCOS data is the mean of several measurements obtained over one day. Different symbols (see figure caption) are used to identify the different origins of those data sets. We earlier (Sect. 3.1) discussed the consistency of our two $(1999,2000)$ photometric systems. Using the few measurements collected in 1999 at coincidental dates, we merged our 1999 photometric system and the one used in Hrivnak et al. (2001) together within the photometric system from Arkhipova et al. (2001). We see (in Fig. 5) that in 2000 our data and Hrivnak's data are perfectly linked. Thus, at least in the $V$ filter our two photometric systems (1999 and 2000) seem to be in good agreement. In Fig. 5 the $Y$-axis is called " $V$ " and not "mag (*) - mag (ref)" as in Figs. 1 and 3. This is because we have added an offset to put the HIPPARCOS, Hrivnak and our data in the Arkipova photometric system.

In Fig. 6 we present the $B$-light curve of HD 179821, resulting from the merging of our own $B$-measurements (collected in 1999 and 2000) together with data from Arkhipova et al. (2001) measurements. In Fig. 7 we present the $U$-light curve of HD 179821, resulting from the merging of our own $U$-measurements (collected in 1999 and 2000) together with data from Arkhipova et al. (2001). Note that we have introduced an offset in our data collected in 2000 so as to get both $U$ light curve maximum (in 1999 and in 2000) at the same level (see Sect. 3.1).

\subsection{Fourier transform analysis}

We performed a Fourier transform analysis on these long term (although irregular and rather inhomogenous) observational sets ( $U, B$ and $V$-light curves). We used the Period98 software (V1.0.4, 1996-1998) from Sperl (1998) that allows prewhitening of selected frequencies. First of all, we applied the Fourier transform analysis on different cuts in the data of the $V$-light curve (displayed in Fig. 5), so as to test the consistency of any results we obtain. Various attempts all lead to the same statement: several periods are present in the resulting Fourier power spectrum whatever the considered sub-sample of data (and whatever the observational origin of these data). Also, there is absolutely no clear feature corresponding to periods lower than 45 days. These facts are also observed from the analysis of $B$ and $U$ data.

We studied the power spectrum of the $V$-light curve (displayed in Fig. 5) in the relevant region for frequency investigation (i.e, for $v$ below 0.025). A main frequency is found at $v_{1}=0.00492\left(P_{1} \sim 203\right.$ days $)$. Important peaks are also present in the low frequencies region: from 0.00024 (which is the length of coverage of the analysed $V$ light curve) to 0.00086 (which is the main frequency we obtain after a first pre-whitening). A few peaks are also present in the period range 103-192 days, and after a second pre-whitening, another frequency comes out: $v_{2}=0.00716\left(P_{2} \sim 140\right.$ days $)$.

However, merging data from different origins may be a source of error when using the Fourier analysis method. For instance, the introduction of HIPPARCOS data in the $V$-light curve can be problematic. Indeed it brings some inhomogeneities due to the specific photometric system of the satellite. Moreover the obvious light variation of HD 179821, from one year to the next one (or from one observational season to the next one) may also be a source of error introducing aliases in the resulting Fourier power spectra. Finally, we cannot be sure about the consistency of the long-term phenomenon ( $v=0.00086)$ detected in the $V$-light curve analysis but if real, its periodicity may be very doubtfull.

In order to avoid all these problems (merging different data and different peak light levels from one year to another one), we also performed a period search on a $V$-light curve slightly modified from Fig. 5. Indeed, for each observational season, we defined a mean level as $\left(V_{\max }-V_{\min }\right) / 2$ and we have corrected by this seasonal mean level all the measurements belonging to the considered season. This method, acting as a powerful filter on very low frequencies, presents the advantage of keeping only the features related to intrinsic pulsations with periods of less than a year. Thus, the long term phenomenon does not appear anymore and does not perturb the frequency analysis. A bimodal pulsation behavior is then found (see Table 1) with two frequencies: $v_{1}=0.00499\left(P_{1} \sim 200\right.$ days $)$ and $v_{2}=0.00719\left(P_{2} \sim 139\right.$ days $)$. Moreover, we can note 


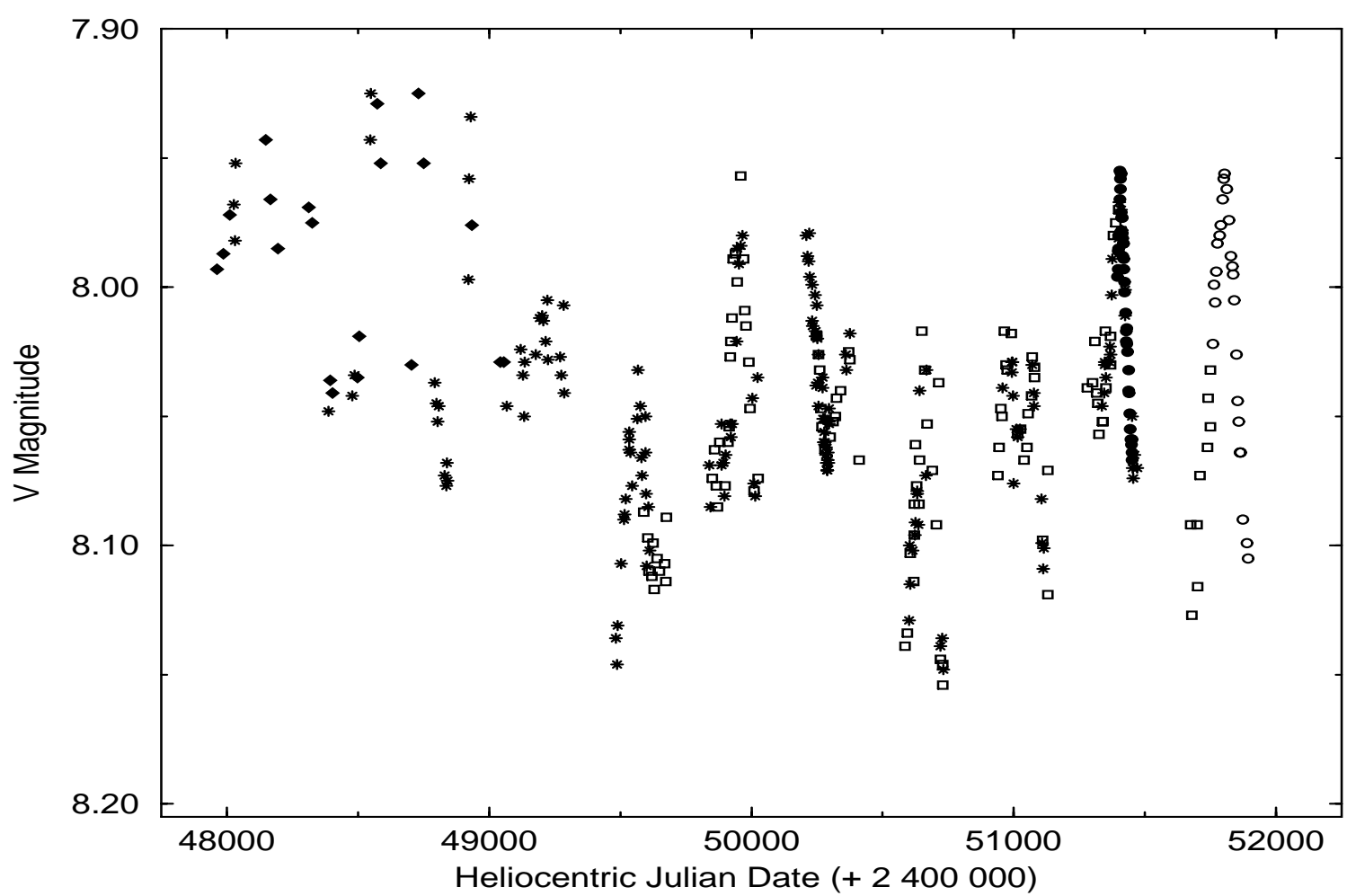

Fig. 5. $V$-filter light curve of HD 179821, resulting from the merging of our own measurements collected in 1999 and 2000 (filled and open circles, respectively) together with the measurements of Arkhipova et al. (2001, stars), Hrivnak et al. (2001, open squares) and HIPPARCOS data (diamonds).

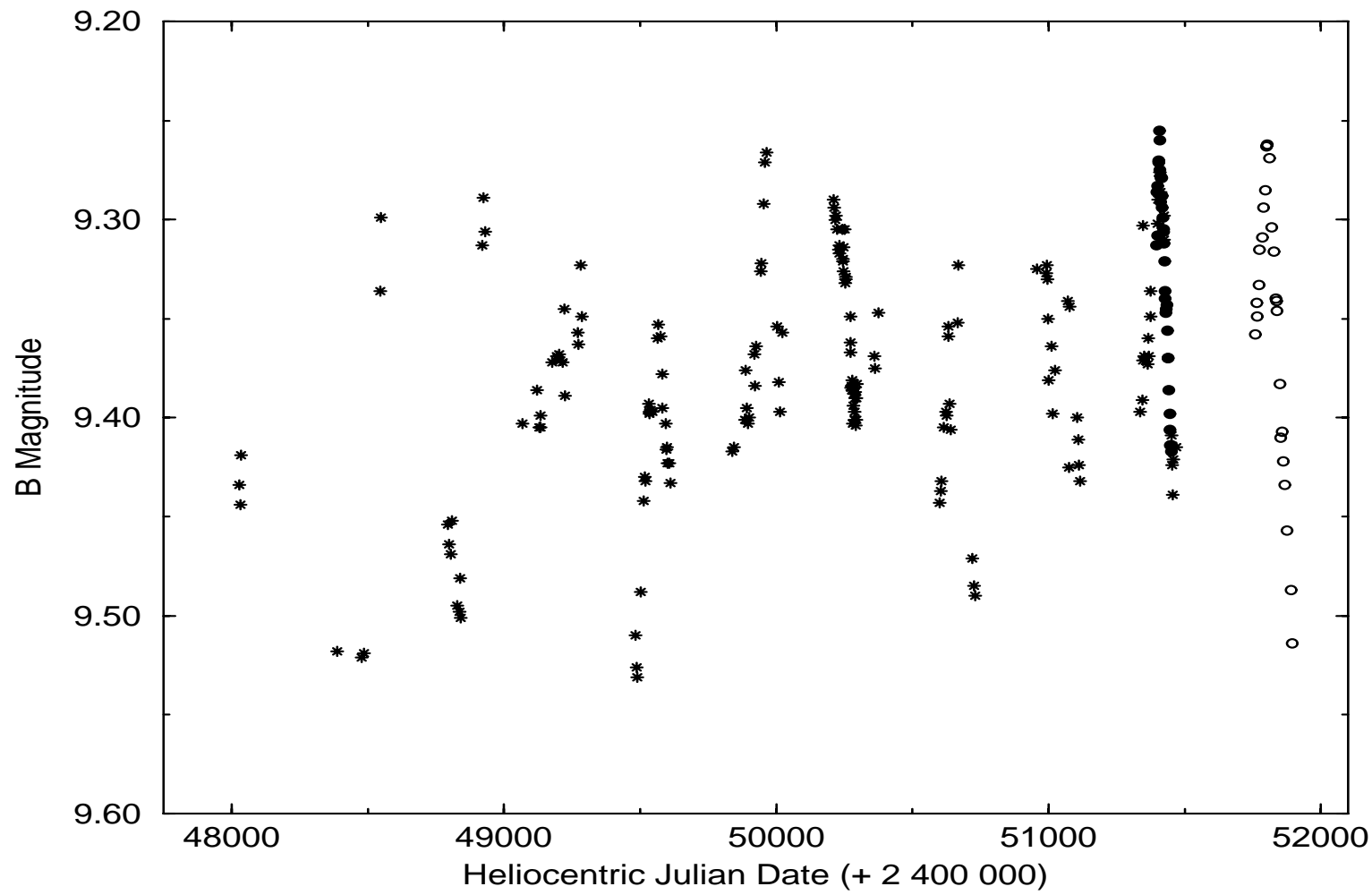

Fig. 6. $B$-filter light curve of HD 179821, resulting from the merging of our own measurements collected in 1999 and 2000 (filled and open circles, respectively) together with the measurements of Arkhipova et al. (2001, stars). 


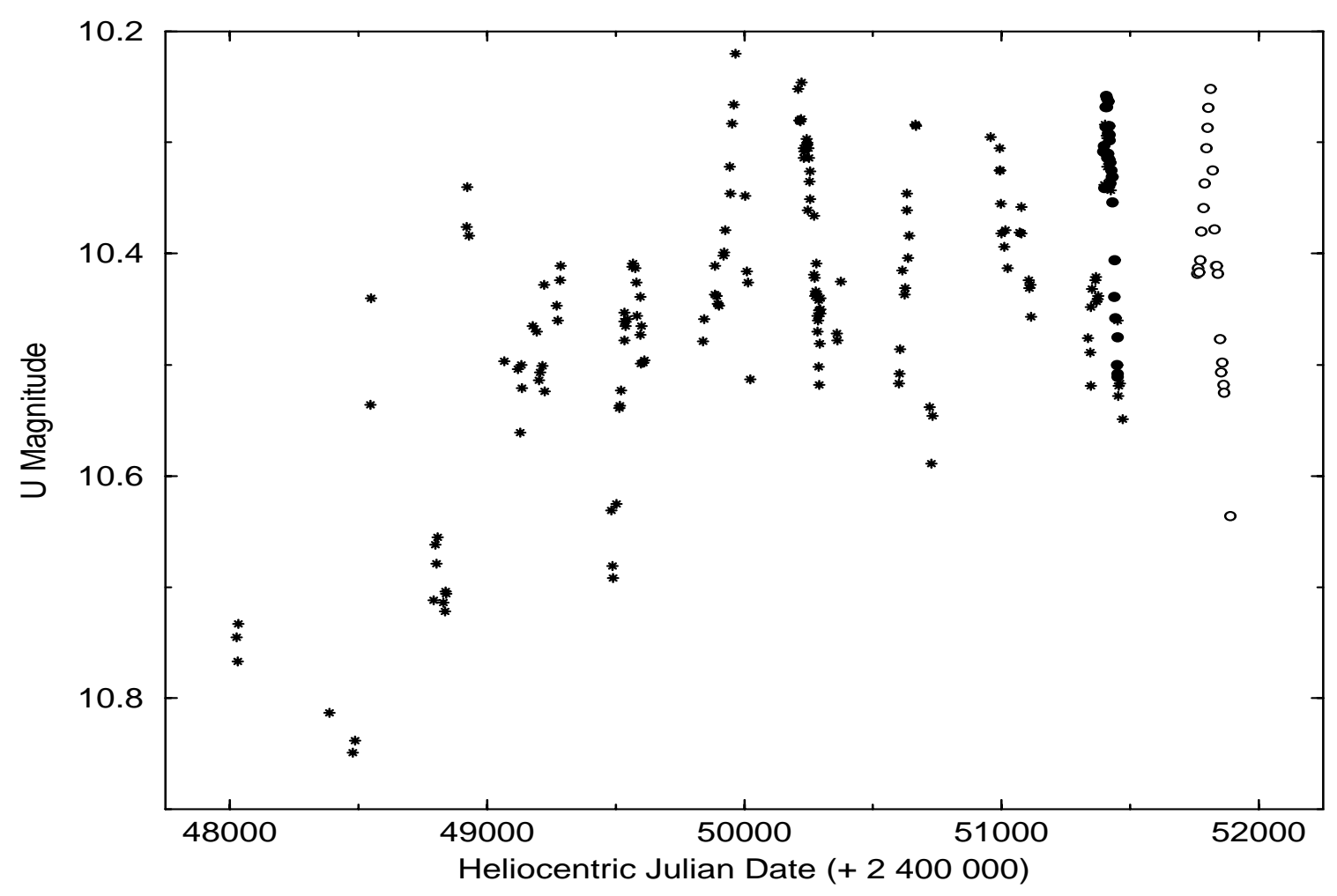

Fig. 7. $U$-filter light curve of HD 179821, resulting from the merging of our own measurements collected in 1999 and 2000 (filled and open circles, respectively) together with the measurements of Arkhipova et al. (2001, stars).

that $\frac{v_{2}}{v_{1}} \simeq \frac{2}{3}$. In this context, $v_{1}$ might be a radial fundamental mode and $v_{2}$ its first overtone. This result was already proposed by Arkhipova et al. (2001). Furthermore, $\frac{v_{2}}{v_{1}} \simeq \frac{2}{3}$ could be also linked to some resonance phenomenon. Another frequency $v_{3}=0.00952$ (about two times $v_{1}$ ) could be linked to a light curve asymmetry. It is the result of the Fourier decomposition of a non-sinusoidal curve. Finally, a third (significant) frequency, $v_{4}=0.00525$ also seems to be present but it is more disputable. This last frequency could be due to the light curve irregularity and notably to the small variations observed in 1993 around HJD = 2449250 (see Fig. 5).

We have also applied the Fourier transform method on the $B$ and $U$-light curves (displayed in Figs. 6 and 7). In the same way as for $V$-data, we have defined for each observational season a mean level and corrected for this seasonal mean level all the measurements belonging to the considered season. The $U$, $B$ and $V$-power spectra we found with this method are given in Fig. 8. However, note that the pulsation frequencies and amplitudes presented in Table 1 are slightly different from the peaks found in Fig. 8. Indeed, in the method we used, each frequency is found after pre-whitening the data set with the previous frequency. Then a theoretical light curve is built with the resulting amplitude and frequency. Finally, through period 98 software those parameters can be very slightly modified (as an adjustement through a least square method process) so as to better fit the light curves.

From the $B$ and $U$-filter data, we find about the same frequencies $\left(v_{1}\right.$ and $\left.v_{2}\right)$ as in the $V$-filter. Note that in the $U$-filter, the $0.0075 \mathrm{c} / \mathrm{d}$ frequency becomes dominant (see Table 1 ). This phenomenon could be due to the fact that $v_{1}$ and $v_{2}$ may be
Table 1. Frequency found in the $U, B, V$-filter power spectra (see Fig. 8). Each frequency $v_{n}$ is found after pre-whitening the data set with the previous frequency $v_{n-1}$. We have adjusted these periods and amplitudes with a least square method to better fit to the light curves (in each filter).

\begin{tabular}{lrr}
\hline \hline Filter & Frequency (c/d) & Amplitude (mag) \\
\hline$V$ & $v_{1}=0.00499$ & $a_{1}=0.031$ \\
& $v_{2}=0.00719$ & $a_{2}=0.017$ \\
& $v_{3}=0.00952$ & $a_{3}=0.018$ \\
& $v_{4}=0.00525$ & $a_{4}=0.012$ \\
$B$ & $v_{1}=0.00480$ & $a_{1}=0.051$ \\
& $v_{2}=0.00712$ & $a_{2}=0.033$ \\
$U$ & $v_{1}=0.00754$ & $a_{1}=0.075$ \\
& $v_{2}=0.00449$ & $a_{2}=0.048$ \\
& $v_{3}=0.00557$ & $a_{3}=0.044$ \\
\hline
\end{tabular}

independent pulsational modes. It could be also a sampling data phenomenon.

In any case, the important variations in the light curves of HD 179821, from one year to another, cannot be satisfactorily explained by a strictly bimodal pulsation. Moreover, with all detected frequencies, it is impossible to obtain a good fit to the light curves. This is probably due to a chaotic behaviour in the pulsation of HD 179821. Furthermore, it is possible that, ten years ago, the $V$ light curve was more chaotic than now. Indeed, the shape of the light curve between the Julian days 2447750 and 2449250 is really not regular (see Fig. 5). Unfortunately, at this epoch, the number of points is not large enough to confirm this effect. 

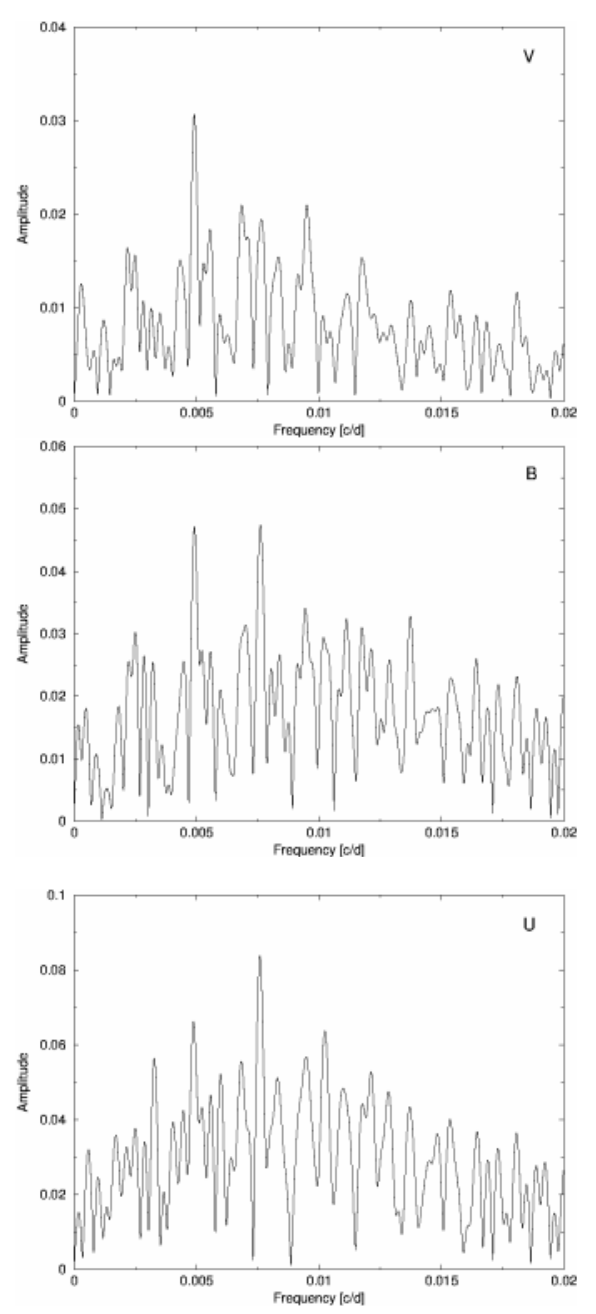

Fig. 8. From bottom to top: $U, B, V$ power spectrum of HD 179821 light curves.

\section{Conclusion}

In this work, we have presented photometric observations of an oxygen-rich evolved star HD 179821. From observations collected at the Haute-Provence Observatory during 1999 and 2000, we found a strong color effect during an almost complete cycle of pulsation with a decreasing photometric amplitude when the filter wavelength is increasing from UV to IR. Typically we have observed a variation of about 0.25 mag in the $U$-filter, while a variation of only about 0.07 mag was observed in the $I$-filter. This effect is due to temperature or opacity variations rather than to radius variations. This is also confirmed by spectroscopic observations that we have obtained for HD 179821 and that we will present in a forthcoming paper. Indeed, we have found that photospheric lines in HD 179821, such as Ba II lines, are relatively stable (in shape and radial velocity) over long intervals of time. Note that this result is very different from observations on the post-AGB star HD 56126, that presents a photospheric velocity variation of about $15 \mathrm{~km} \mathrm{~s}^{-1}$ over few days (Barthès et al. 2000).

Considering all the available photometric data for HD 179821 (HIPPARCOS, Arkhipova et al. 2001; Hrivnak et al. 2001), we have constructed long term (over about ten years), relatively complete $U, B, V$-light curves. With all these data, we have looked for the HD 179821 pulsation period(s) with a Fourier transform analysis. In $U, B$ and $V$-filters, we have always found two main periods. Because there are more data sets in the $V$-filter than in the other filters, we have concluded that the periods $P_{1}=200$ days and $P_{2}=139$ days found in the $V$-filter are the most accurate results that we can produce. As we found about the same periods (maximum 20 days of difference) with data from the other filters, we can be sure that $P_{1}$ and $P_{2}$ are real. Note that if we try to fit the light curve with $P_{1}$ and $P_{2}$ or with more periods (for example, using the long period $P \simeq 1000$ days), we do not get a definitive result. HD 179821 is probably not strictly periodic and may have a chaotic pulsational behavior.

On the basis of the few post-AGB stars that have been studied for variability (see Arkhipova et al. 2000; Hrivnak et al. 2001), a long period ( $P>130$ days, like the one we found in HD 179821) is generally associated with a pulsation amplitude higher than $0.2 \mathrm{mag}$. However in these previous studies, two post-AGB stars have been reported with the same kind of pulsation amplitude and period as HD 179821: V887 Her (Arkhipova et al. 2000) and IRAS 07430+1115 (Hrivnak et al. 2001). Nevertheless, for a low mass star, non-linear radiative pulsation models are not able to reproduce long periods ( $P>130$ days) with low amplitudes $(\Delta m \simeq 0.2)$ such as the ones found for HD 179821 (A. Fokin, private communication). Pulsation models could consider HD 179821 as a massive star, but then convection (of super-massive stars) has to be taken into account. Unfortunatly, the best models available up to now do not take into account the convection which can reduce the light amplitude and may possibly change the pulsational behavior of the model. Thus as a next step forward, pulsation models including convection, on one hand, and long-term observations for a relevant sample of post-AGB stars (together with period analysis), on the other hand, will help to provide a definitive statement about the nature of HD 179821.

Acknowledgements. The authors are grateful to F. Daudé, student at University of Montpellier, for his help in reduction of the 1999 data. This research has been partially funded by specific financial support from PNPS/INSU. It has made use of the soft Period98 (V1.0.4, 19961998) developed by M. Sperl at Institute of Astronomy, University of Vienna, the SIMBAD database, operated at CDS, Strasbourg, France, and the NASA ADS service, USA. We are much indebted to the referee, Dr. B. Hrivnak, for his critical reading of this paper and valuable advice.

\section{References}

Arkhipova, V. P., Ikonnikova, N. P., Noskova, R. I., Sokol, G. V., \& Shugarov, S. Yu. 2001, Astron. Lett., 27, 156

Arkhipova, V. P., Ikonnikova, N. P., Noskova, R. I., \& Sokol, G. V. 2000, Astron. Lett., 26, 609

Barthès, D., Lèbre, A., Gillet, D., \& Mauron, N. 2000, A\&A, 359, 168

Busso, M., Lambert, D. L., Beglio, L., et al. 1995, ApJ, 446, 775

Gledhill, T. M., Yates, J. A., \& Richards, A. M. S. 2001, MNRAS, 328, 301

Gledhill, T. M., \& Takami, M. 2001, MNRAS, 328, 266 
Hawkins, G. W., Skinner, C. J., Meixner, M. M., et al. 1995, ApJ, 452, 314

Hrivnak B. J. 2001, in Torun Workshop "post-AGB Objects as a Phase of Stellar Evolution”, ed. R. Szczerba, \& S. K. Górny, Astroph. Space Sci. Library, 265 (Kluwer Academic Pub), 101

Josselin, E., \& Lèbre, A. 2001, A\&A, 367, 826

Jura, M., \& Werner, M. W. 1999, ApJ, 525, L116

Miller, G. E., \& Scalo, J. M. 1979, ApJS, 41, 513

Molster, F. J., Waters, L. B. F. M., Tielens, A. G. G. M., \& Barlow, M. J. 2002, A\&A, 382, 184

Reddy, B. E., \& Hrivnak, B. J. 1999, ApJ, 117, 1834
Sperl, M. 1998, Period98 soft V1.0.4, Institute of Astronomy, University of Vienna

Thèvenin, F., Parthasarathy, M., \& Jasniewicz, G. 2000, A\&A, 359, 138

Van Winckel, H., Waelkens, C., \& Waters, L. B. F. M. 1996, A\&A, 306, L37

Zacs, L., Klochkova, V. G., Panchuk, V. E., \& Spelmanis, R. 1996, MNRAS, 282, 1171

Zalewski, J. 1993, Acta Astron., 43, 431

Zuckerman, B., \& Dyck, H. M. 1986, ApJ, 311, 345 